\title{
Dental Students' Emotions, Knowledge, Awareness And Learning Intention On The Applications of Nanotechnology In Dentistry
}

Erdal Senocak ( $\nabla$ erdalsenocak60@gmail.com )

Tokat Gaziosmanpaşa University

Işıl Sarıkaya

Tokat Gaziosmanpaşa University

Hüseyin Hatırlı

Tokat Gaziosmanpaşa University

Alem Coşgun

Tokat Gaziosmanpaşa University

\section{Research Article}

Keywords: Educational Measurement, Undergraduate Dental Education, Assessment, Applications of Nanotechnology

Posted Date: February 3rd, 2022

DOI: https://doi.org/10.21203/rs.3.rs-1255813/v1

License: (c) (i) This work is licensed under a Creative Commons Attribution 4.0 International License. Read Full License 


\section{Abstract}

Background: Dentistry includes vast applications of nanotechnology from preventive dentistry to diagnostics. However, we have limited knowledge about dentistry students' perceptions of the emerging applications of nanotechnology in dentistry. This study aims to reveal a group of dental students' knowledge, emotions, awareness and learning intentions on the emerging applications of nanotechnology in dentistry.

Methods: This study was carried out through a quantitative research design. A questionnaire including four parts was administered to a total of 269 dental students from different grade levels. Data were summarized using descriptive statistics and the chi-square test. All statistical analysis were done by using the SPSS Version 22.

Results: Findings of the dataset indicated that most of the participants had heard some or a lot about nanotechnology. Also, they displayed positive emotions toward nanotechnology. Almost $60 \%$ of participants stated that they know what nanotechnology means, whereas more than $70 \%$ of them reported that they don't know how nanotechnology works. Findings also showed that as the grade level increased, more students became aware of the applications of nanotechnology. In addition, the participants thought that they needed to get more information about the applications of nanotechnology in dentistry education and in future dental practices.

Conclusion: The results of the study revealed that dental students were familiar with nanotechnology and displayed positive emotions towards nanotechnology. Also they desired to learn more information about the applications of nanotechnology. In addition, findings showed that as the grade level increased, more students became aware of the applications of nanotechnology.

\section{Background}

Nanotechnology is defined as the design, characterisation, production and application of structures, devices and systems by controlling shape and size at nanometre scale. ${ }^{1}$ At nanoscale (1 to $100 \mathrm{~nm}$ ), the mechanical, electrical, optical, thermal, and magnetic properties of a nanoparticle (NP) are different from those of its bulk material. ${ }^{2}$ Nanotechnology has been utilized in various research fields including electronics, cosmetics and medicine., ${ }^{3,4}$ The unique chemical, biological and physical properties of NPs make them highly desirable in a variety of applications. ${ }^{5}$ Novel properties of NPs facilitate their application in different areas. For example, carbon nanotubes (CNT) have specific properties such as high tensile strength and conductivity, which thereby have the potential to be used in several applications in engineering and materials science. ${ }^{6}$ Nanotechnology is also utilized for nanodentistry where NPs are used to improve durability of dental fillings, prevent oral diseases or restore damaged dental tissues. ${ }^{7-9}$ Today, this branch of technology has a wide variety of applications in different subspecialties of dentistry from oral diseases diagnosis to restoration by using nanostructured materials. ${ }^{10-15}$ The recent advances in nanodentistry promise to bring a paradigm shift in dentistry. A variety of new dental products are on 
the market daily, from implants to oral hygiene products based on nanoscale properties. ${ }^{16}$ Also, the number of products for various dental applications is expected to increase remarkably in the near future. $^{12}$

In accordance with the developments in nanotechnology, dentistry schools are offering some courses or integrating nanotechnology in their current curriculums for raising the awareness of the field of nanodentistry and its various uses. However, related literature has limited research revealing the impact of this effort or to understand nanotechnology awareness of dentistry students. ${ }^{17,18}$ As for Turkey, there isn't any study capturing dentistry students' reactions to nanotechnology such as awareness, knowledge or attitudes. The present study mainly aimed to reveal dentistry students' awareness of the emerging applications of nanotechnology in dentistry. Besides, students' knowledge of, emotions about and learning intention to nanotechnology were also examined.

Several studies have examined the public's, teachers' or experts' perceptions of nanotechnology ${ }^{19-22}$ because stakeholders' reactions to a new technology are the key factors, possibly influencing its development. Kohler and Som stressed that public opinion has a strong impact on the progress of a new technology. ${ }^{23}$ Although rapid developments in nanodentistry are expected, there is limited knowledge about how familiar dental students with nanotechnology, emotional reactions to this technology, or awareness levels of its applications in dentistry. Therefore, this study aimed to take a step towards realizing importance of examining dentistry students' knowledge, emotions and awareness towards the emerging applications of nanotechnology in dentistry.

\section{Methods}

\section{Sample}

In 2021, an online survey was administered to a randomly selected sample of dentistry students attending their second, third, fourth and fifth years to assess their perceptions of nanotechnology and its applications in dentistry. Students have volunteered to participate the study through an online survey and their identity anonymously. First year students were not involved in the study by assuming they have little or no knowledge about nanotechnology applications in dentistry. The reason that led us to this assumption is that first year students did not attend a course on dental materials or any dental clinic practice.

\section{Questionnaire}

Data were collected through a questionnaire consisted of four parts. Part 1 included three items questioning participants' emotions about nanotechnology. Part 2 included three items that assessed participants' knowledge of nanotechnology. Part 3 aimed to reveal participants' awareness of nanotechnology applications in dentistry through 12 items. These items are collected under three 
categories, respectively preventive dentistry, therapeutic dentistry and diagnostic dentistry. For example, an item under the preventive dentistry section was stated, "Silver nanoparticles are used in dentistry as an antimicrobial" and was coded "I have no idea, I agree, I disagree". Students first read the statement and chose one of the codes which reflected their ideas best. As for Part 4, it included three items questioning participants' learning intention about nanotechnology applications in dentistry. For example, the first item was stated, "I need more information about the applications of nanotechnology in dentistry", and was coded "Strongly Agree, Somewhat Agree, Slightly Agree, Disagree".

The questionnaire was developed in four stages. First, the authors reviewed the studies on nanotechnology perceptions of the general public. As mentioned above, the first and second part of the questionnaire aimed to reveal participants' emotions and knowledge of nanotechnology. These parts of the questionnaire were created by modifying or refining items from widely used surveys to measure

public's perceptions of nanotechnology. ${ }^{22,24}$ Second, a group of experts came together to construct the Part 3 of the questionnaire. The first author wrote some items reflecting the applications of nanotechnology in dentistry. Then items were reviewed by three content experts from various disciplines at the faculty of dentistry. The experts decided to delete or revise some items, they also added new items to the questionnaire. Then they collected the items in three categories (preventive, therapeutic and diagnostic). Overall, Part 3 included 12 items related to applications of nanotechnology in dentistry under three categories. Third, the questionnaire with three parts was piloted by 12 randomly selected students and students were interviewed. Then the questionnaire was further revised based on students' feedback. Some students suggested adding a new part to the questionnaire related to the intention for information about nanotechnology applications in dentistry. In accordance with the suggestion, a new part added to the questionnaire. This part included three items related to motivation for learning nanotechnology applications. Lastly, the final version of the questionnaire was implemented to 93 dentistry students to calculate internal consistency reliability. Internal consistency reliability in terms of Cronbach's alpha was calculated as 0.85 . As a result, a questionnaire was constituted including 21 items and four parts with high reliability and content validity.

\section{Statistical analysis}

Both the percentage frequency distributions and the chi-square $(x 2)$ test were used to examine the relationships between participants' grade levels and their knowledge, attitudes, awareness and learning intention on the emerging applications of nanotechnology in dentistry. The significance level for the analysis was set at $p \leq 0.05$. The data were statistically analyzed and represented in tables. Descriptive statistics were used to show the distribution of values in the dataset. The chi-square test as a nonparametric statistic was preferred because the items in the questionnaire included categorical variables. Non-parametric statistics have some assumptions, such as 'the data don't follow a normal distribution' or 'the data that are ordinal'.

\section{Results}


The findings of the study were categorized into two groups: descriptive statistics and non-parametric statistics.

Descriptive statistics were used to provide simple summaries about the sample and the measures. A sample of 269 dentistry students were reached in the study to reveal their perceptions of nanodentistry and its applications in dentistry. The sample included students from different grade levels: 2 nd year (39\%, $106)$, 3rd year $(26 \%, n=70)$, 4th year $(16 \%, n=43)$, and 5 th year $(18 \%, n=50)$. First part of the questionnaire included three items questioning students' emotions about nanotechnology. Table 1 displays the distribution of responses of participants to the items in Part 1 according to their grade levels.

Table 1

Descriptive statistics related to the items in Part 1

\begin{tabular}{|llllll|}
\hline \multicolumn{5}{l}{ Grade Levels } \\
\hline
\end{tabular}

Table 1 represents the percentages of students' choices who were in different grades with respect to the items in the first part of the questionnaire in terms of their emotions about nanotechnology. Table 1 shows that most of the participants had heard some or a lot about nanotechnology before, however, there were no students who had heard nothing about nanotechnology. Table 1 also displays that just a small percentage of the students were worried about nanotechnology when they heard something about it. 
Mostly they had a hopeful feeling towards nanotechnology. In addition, students' perceptions of the risks and benefits of nanotechnology were investigated. As seen from Table 1, the majority of the students felt that the benefits outweigh the risks of nanotechnology.

As for the findings on students' knowledge of nanotechnology, the questionnaire included three items questioning the students' nanotechnology knowledge. These items addressed the meaning of nanotechnology, how nanotechnology works, and the size of nanoscale. The participants responded to these items with 'Yes' or 'No'. As seen from Table 2, students from different academic grades mostly answered 'Yes' to the items attributing the meaning of nanotechnology and the size of nanoscale. On the contrary, most of the participants answered as "No" the item questioning how nanotechnology works. This finding gives hints that most of the students did not exactly have essential ideas of nanotechnology such as how matter behaves differently at nanoscale from their macroscopic counterparts or which kind of interactions are dominant at nano- and atomic-scale.

Table 2

Descriptive statistics related to the items in Part 2

\begin{tabular}{|c|c|c|c|c|c|}
\hline \multicolumn{6}{|c|}{ Grade Levels } \\
\hline & Items & 2nd (\%) & 3rd (\%) & 4th (\%) & 5th (\%) \\
\hline \multirow[t]{9}{*}{ Nanotechnology Knowledge } & \multicolumn{5}{|c|}{ Can you explain what nanotechnology means? } \\
\hline & Yes & 67.9 & 67.1 & 67.4 & 64 \\
\hline & No & 32.1 & 32.9 & 32.6 & 36 \\
\hline & \multicolumn{5}{|c|}{ Can you explain how nanotechnology works? } \\
\hline & Yes & 28.3 & 28.6 & 18.6 & 30 \\
\hline & No & 71.7 & 71.4 & 81.4 & 70 \\
\hline & \multicolumn{5}{|c|}{ Can you explain how many nanometers there are in a meter? } \\
\hline & Yes & 52.8 & 50 & 60.5 & 64 \\
\hline & No & 47.2 & 50 & 39.5 & 36 \\
\hline
\end{tabular}

The Chi-Square test was used to examine if there were significant relationships among students' grade levels and their reactions to the items in Part 2 and 3 of the questionnaire. The Chi-Square test results showed that there were no statistically significant differences between the students from different grades in terms of their reactions to the items in Part 2 and 3 at $p=.05$ significance level. This means there isn't a statistical relationship between the students' grades and their emotions about and knowledge of nanotechnology.

The third part of the questionnaire includes 12 items under three categories. These items contain statements about the emerging applications of nanotechnology in dentistry. Table 3 shows that second 
and third grade students mostly marked the option "no idea" about the applications of nanotechnology for all items, while fourth and fifth grade students marked the option "I agree" except for two items (There are toothpastes and mouthwashes in the market which contain nanoparticles such as titanium dioxide; Nanoparticles are used in the diagnosis of oral cancer lesions at early stages). Table 3 also indicates that for almost all items, as the grade level of the participants increases, their answers get closer to the option "I agree". For example, "I agree" response rates of second, third, fourth and fifth grade students for the third item under the preventive dentistry category are as follows, $30.2 \%, 45.7 \%, 79.1 \%, 94 \%$. In another example, for the statement in the fifth item under the category of therapeutic dentistry, $33 \%$ of the secondyear students preferred the option "I agree", while $76 \%$ of the fifth-year students preferred the option "I agree". These findings refer that as the grade level increases, the awareness of the students about the applications of nanotechnology in dentistry increases. However, it is seen that the awareness of the participants about some applications of nanotechnology in dentistry is low. 
Table 3

Descriptive statistics related to the items in Part 3

\section{Grade Levels}

Items $\quad$ 2nd (\%) $\quad 3 r d(\%) \quad$ 4th (\%) $\quad$ 5th (\%)

Preventive Silver nanoparticles are incorporated into dental composites to inhibit the bacterial dentistry growth.

\begin{tabular}{lllll} 
I agree & 13.2 & 28.6 & 44.2 & 72 \\
\hline I don't agree & - & 1.4 & 2.3 & 6 \\
\hline No idea & 86.8 & 70 & 53.5 & 22
\end{tabular}

There are toothpastes and mouthwashes in the market which contain nanoparticles such as titanium dioxide.

\begin{tabular}{lllll|}
\hline I agree & 28.3 & 12.9 & 23.3 & 46 \\
\hline I don't agree & 1.9 & 2.9 & 2.3 & 2 \\
\hline No idea & 69.8 & 84.3 & 74.4 & 52 \\
\hline
\end{tabular}

Nanoparticles are used to prevent tooth decay or reduce its progression.

\begin{tabular}{lllll}
\hline I agree & 30.2 & 45.7 & 79.1 & 94 \\
\hline I don't agree & - & - & - & - \\
\hline No idea & 69.8 & 54.9 & 20.9 & 6
\end{tabular}

Nanoparticles are utilized in acrylic resins to show improved antimicrobial and antifungal effects.

\begin{tabular}{lllll}
\hline I agree & 34.9 & 32.9 & 55.8 & 68 \\
\hline I don't agree & 1.9 & 2.9 & 2.3 & 6 \\
\hline No idea & 63.2 & 64.3 & 41.9 & 26
\end{tabular}

Therapeutic Nanoparticles are used for controlled drug delivery in the treatment for periodontal dentistry diseases.

\begin{tabular}{|lllll|}
\hline I agree & 27.4 & 24.3 & 53.5 & 52 \\
\hline I don't agree & 3.8 & - & - & 2.0 \\
\hline No idea & 68.9 & 75.7 & 46.5 & 46 \\
\hline
\end{tabular}

Nanoparticles are used to increase the esthetic, mechanical and physical properties of composite resins and glass ionomer-based restorative materials.

$\begin{array}{lllll}\text { I agree } & 48.1 & 42.9 & 74.4 & 84 \\ \text { I don't agree } & 1.9 & - & - & -\end{array}$




\section{Grade Levels}

No idea

50.0

57.1

25.6

16

Nanoparticles are used for dental implant coating to promote the implants in terms of mechanically and biologically.

\begin{tabular}{lllll|}
\hline I agree & 44.3 & 40 & 79.1 & 84 \\
\hline I don't agree & - & - & - & - \\
\hline No idea & 55.7 & 60 & 20.9 & 16
\end{tabular}

Nanoparticles are used to increase the bond strength of cements and dental adhesives.

\begin{tabular}{lllll}
\hline I agree & 48.1 & 35.7 & 72.1 & 74 \\
\hline I don't agree & - & - & - & 2.0 \\
\hline No idea & 50.0 & 64.3 & 27.9 & 24 \\
\hline
\end{tabular}

Nano-hydroxyapatite is used for the treatment of dentin sensitivity.

\begin{tabular}{lllll}
\hline I agree & 33.0 & 32.9 & 48.8 & 76 \\
\hline I don't agree & 1.9 & - & 2.3 & 4.0 \\
\hline No idea & 65.1 & 67.1 & 48.8 & 20
\end{tabular}

Diagnostic Nanoparticles are used in the diagnosis of oral cancer lesions at early stages. dentistry

\begin{tabular}{lllll}
\hline I agree & 26.4 & 21.4 & 32.6 & 30 \\
\hline I don't agree & 0.9 & - & - & 12 \\
\hline No idea & 72.6 & 78.6 & 67.4 & 58
\end{tabular}

Nanoparticles can be used as an auxiliary diagnostic material for dental caries diagnosis methods.

\begin{tabular}{lllll|}
\hline I agree & 48.1 & 30 & 51.2 & 68 \\
\hline I don't agree & - & 2.9 & - & - \\
\hline No idea & 51.9 & 67.1 & 48.8 & 32 \\
\hline \multicolumn{4}{l}{ Nanotechnology } & promises the diagnosis of dental diseases at molecular level. \\
\hline I agree & 49.1 & 30 & 51.2 & 62 \\
\hline I don't agree & - & 4.3 & 2.3 & - \\
\hline No idea & 50.9 & 65.7 & 46.5 & 38 \\
\hline
\end{tabular}


The Chi-Square test was used to examine if there were significant relationships among students' grade levels and their reactions to the items in Part 3 of the questionnaire. The test results showed that there were statistically significant differences among the students from different grades in terms of items in Part 3 at $p=.05$ significance level for all items. These means there are statistical relationships between the students' grade levels and their perceptions of nanotechnology applications in dentistry. Namely, as the grade level increases, students' awareness of the use of nanotechnology in dentistry increases significantly.

In Part 4 of the questionnaire, students were asked about how much they intended to learn about nanotechnology applications in dentistry through three items. Table 4 shows that most of the students from all grades agree that they want to learn more about the applications of nanotechnology in dentistry and be included more information about the applications of nanotechnology in dentistry in the dental curriculum. Findings also show that students intend to continue to learn about applications of nanotechnology in dentistry in their future dental practice. In addition, according to the findings presented in Table 4, fourth and fifth graders are less eager to learn about the dental applications of nanotechnology than other students. Overall, students expressed their willingness to learn more about nanotechnology during their undergraduate education and in future dental practice. 
Table 4

Descriptive statistics related to the items in Part 4

\section{Grade Levels}

$\begin{array}{lllll}\text { Items } & \text { 2nd (\%) } & \text { 3rd (\%) } & \text { 4th (\%) } & \text { 5th (\%) }\end{array}$

I need more information about the usage of nanotechnology in dentistry.

\begin{tabular}{lllll} 
Strongly Agree & 93.4 & 91.3 & 81.4 & 76.0 \\
\hline Somewhat Agree & 5.7 & 5.9 & 18.6 & 20.0 \\
Slightly Agree & 0.9 & - & - & 2.0 \\
Disagree & - & 2.9 & - & 2.0
\end{tabular}

I would prefer to be included more information about the applications of nanotechnology in dentistry in the dental curriculum.

\begin{tabular}{|lcccc|}
\hline Strongly Agree & 81.1 & 75.4 & 79.1 & 58.0 \\
\hline Somewhat Agree & 18.9 & 21.7 & 16.3 & 36 \\
\hline Slightly Agree & - & - & 4.7 & 4.0 \\
\hline $\begin{array}{l}\text { Disagree } \\
\begin{array}{l}\text { I would like to continue to learn about applications } \\
\text { practice. }\end{array}\end{array}$ & - & -- & 2.0 \\
\hline $\begin{array}{l}\text { Strongly Agree } \\
\text { Somewhat Agree }\end{array}$ & 84.0 & 82.6 & 60.5 & 52.0 \\
\hline Slightly Agree & 16.0 & 14.5 & 30.2 & 36.0 \\
\hline Disagree & - & - & 7.0 & 4.0 \\
\hline
\end{tabular}

Chi-square test was utilized to examine the relationships between participants' grade levels and three items in Part 4 at a $p<0.05$ level of significance. According to the chi-square statistics, all items had statistically significant relationships with students' grade levels. This means that there was a significant difference between students' grades and their intention to learn about applications of nanotechnology in dentistry. Second and third graders were more motivated to learn about applications of nanotechnology in dentistry than fourth and fifth graders.

\section{Discussion}

In this study, cooperated with a group of dentistry students to reveal their perspectives through a questionnaire with 21 items under four parts: emotions about nanotechnology, nanotechnology knowledge, awareness of nanotechnology applications in dentistry and learning intention about nanotechnology applications. The findings indicated that dental students recognized themselves to be 
familiar with nanotechnology. More than $50 \%$ of participants heard some or a lot about nanotechnology before. The findings also revealed that students displayed positive emotions toward nanotechnology. Just a few students were worried when they heard about nanotechnology. Similarly, participants had little concern about risks of nanotechnology. Only 3 out of 269 students stressed the risks outweigh the benefits. We couldn't find any research in literature that investigated the emotions of dental students about nanotechnology. Therefore, the findings of the study have a potential to understand prospective dentists' emotional reactions to nanotechnology. Determining dental students' emotions is also important because such feelings may have a potential influence on prospective dentists' perceptions related to nanotechnology applications. The chi-square test results showed that students' grade level wasn't associated with students' emotions about nanotechnology. Namely, students' class level isn't an important variable that influences students' emotions about nanotechnology.

The study also investigated the participants' knowledge of nanotechnology. Three items were used to survey students' nanotechnology knowledge. The data showed that most students from all classes approved themselves to explain what nanotechnology means and how many nanometers there are in a meter. Surprisingly, most of the participants couldn't explain how nanotechnology works. These findings show that dental students had a fair knowledge of nanotechnology. They know what nanotechnology is but couldn't explain its fundamental concepts in detail. As for the chi-square test results, there wasn't any statistically difference between students' grades and their nanotechnology knowledge. In other words, participants' nanotechnology knowledge wasn't associated with their grade levels.

Another concern of the study was to reveal dental students' awareness towards the emerging applications of nanotechnology in dentistry. As pointed out before, 12 items were formed under three categories to elicit participants' awareness towards nanodentistry. The data about students' awareness of nanodentistry may be divided into two sections: findings about second and third grades, and fourth and fifth graders. Second and third graders qualified themselves to be not aware of the applications of nanotechnology in dentistry. $50 \%$ or more than $50 \%$ of this group of students marked the option "no idea" as answer for all items questioning nanodentisty applications from different aspects. On the contrary, high percentage of fourth and fifth graders marked the option "yes" as the answer for most of the items. They especially referred to themselves to be unfamiliar with the items questioning the applications of nanoparticles in toothpastes and the diagnosis of oral cancer lesions. These findings suggest that dental students especially in the beginning years had limited awareness about the applications of nanotechnology in dentistry, but students improved their awareness in the following years. The chi-square test results confirm these findings. Students with upper grades were significantly more aware than the students with lower grades towards the applications of nanotechnology. In accordance with these findings, it can be concluded that the academic level is an important factor that shapes dental students' awareness towards nanodentistry. There were some studies in literature focused dentistry students' awareness towards nanoparticles incorporation in dental materials. ${ }^{17,18,25}$ The findings of these studies also revealed that grade level was a significant factor affecting students' awareness towards nanodentistry applications. We thought that the reason of upper class students' high level awareness 
may be clinical practices. Students in fourth and fifth classes are involved in clinical practices where they have the opportunity to use dental materials. During the clinical practices, they get some opportunities to learn and use any kind of applications of materials in dentistry.

Lastly, students' intention to learn about applications of nanotechnology in dentistry with respect to different aspects were assessed. The data showed that the vast majority of participants intended to learn about the usage of nanotechnology in dentistry, desire to be included more information about the applications of nanotechnology in dentistry in the dental curriculum, and to continue to learn about applications of nanotechnology in dentistry in the future. However, the chi-square test results and descriptive statistics pointed that upper grade students were less motivated than other students (second and third graders) to learn about applications of nanotechnology in dentistry. We suggested that the reason of upper grade students' lower motivation than other students may be limited usage of the emerging applications of nanotechnology in clinical practices. Traditional dental applications are still used commonly for prospective dentists' clinical practices in dental schools. Therefore, students may gradually lose their attention to learn the emerging applications in dentistry such as nanotechnology.

\section{Conclusion}

In summary, this study examined a group of dental students and reported findings from a survey regarding their emotions, knowledge, awareness, and learning intention on the emerging applications of nanotechnology in dentistry. The study also examined the influence of participants' grade levels on their perceptions. Based on the findings of the study, it can be concluded that dental students are familiar with nanotechnology, positive and hopeful about nanotechnology. In addition, although they have a fair knowledge of nanotechnology, they need more information to explain its fundamental concepts in detail. Besides, students especially from lower grades clearly expressed their desire for more information about the application of nanotechnology in dentistry. However, this group of students recognized themselves not to be aware of the applications of nanotechnology in dentistry. According to the findings of the study, it may be concluded that prospective dentists may interact with learning environments covering the core concepts of nanotechnology which explain how nanotechnology works and give more opportunity to get information about the emerging applications of nanotechnology in dentistry in order to prepare them for the future. We also concluded that the emerging applications in dentistry may be used as much as possible in clinical practices in dentistry schools in order to motivate students to learn about them.

\section{Abbreviations}

nm: nanometre

NP: Nanoparticle

CNT: Carbon nanotubes 


\section{Declarations}

Ethics approval and consent to participate: All methods were performed in accordance with the guidelines and regulations contained in the Declaration of Helsinki. This study was approved by the Tokat Gaziosmanpaşa University Human Ethics committee (approval number 08/01-23) and all participants gave their written informed consent.

Consent for publication: Not applicable.

Availability of data and materials: The data analysed in this study are available from the corresponding author on reasonable request, subject to ethical approval.

Competing interests: The authors declare that they have no competing interests.

Funding: The study was self-funded. No public or private funding source was used for this research.

Authors' contributions: All the authors contributed to the design and implementation of the research. I.S., H.H. and A.C. implemented the questionnaire. E.Ş. analyzed the findings. All the authors discussed the results and commented on the manuscript.

Acknowledgements: Authors would like to thank all the participants for taking part in this study.

\section{References}

1. The Royal Society \& The Royal Academy of Engineering. Nanoscience and nanotechnologies: opportunities and uncertainties. London: The Royal Society; 2004.

2. Sheetza T, Vidalb J, Pearsonc TD, Lozano K. Nanotechnology: Awareness and societal concerns. Tech Soc. 2005;27:329-345.

3. Manke A, Wang L, Rojanasakul Y. Mechanisms of nanoparticle-induced oxidative stress and toxicity. BioMed Res International. 2013;1:1-15.

4. Bayda S, Adeel M, Tuccinardi T, Cordani M, Rizzolio F. The history of nanoscience and nanotechnology: From chemical-physical applications to nanomedicine. Molecules. 2019;25:112126.

5. Jandt KD, Watts DC. Nanotechnology in dentistry: Present and future perspectives on dental nanomaterials. Dent Mat. 2020;36(11):1365-1378.

6. Stella GM. Carbon nanotubes and pleural damage: perspectives of nanosafety in the light of asbestos experience, Biointer Phases. 2011;6(2):1-17.

7. Abou Neel EA, Bozec L, Perez RA, Kim HW, Knowles JC. Nanotechnology in dentistry: prevention, diagnosis, and therapy. Int J Nanomed. 2015;10:6371-6394.

8. AlKahtani RN. The implications and applications of nanotechnology in dentistry: A review. The Saudi Dent J. 2018;30(2):107-116. 
9. Elkassas D, Arafa A. The innovative applications of therapeutic nanostructures in dentistry. Nanomed: Nanotech Biology Med. 2017;13(4):1543-1562.

10. Agnihotri R, Gaur S, Albin S. Nanometals in dentistry: Applications and toxicological implications-a systematic review. Biolog Trace Element Res. 2020:197(1) 70-88.

11. Bhardwaj A, Bhardwaj A, Rao RN. Nanostructures in dentistry: In diagnosis, drug delivery and oral cancer therapy, and their biocompatibility. In: Chaughule R. (eds) Dental Applications of Nanotechnology. Springer, Cham. 2018.

12. Khurshid Z, Zafar M, Qasim S, Shahab S, Naseem M, AbuReqaib A. Advances in nanotechnology for restorative dentistry. Materials. 2015;8:717-731.

13. Lubojanski A, Dobrzynski M, Nowak N, Rewak-Soroczynska J, Sztyler K, Zakrzewski W, Dobrzynski W, Szymonowicz M, Rybak Z, Wiglusz K, Wiglusz RJ. Application of Selected Nanomaterials and Ozone in Modern Clinical Dentistry. Nanomaterials. 2021;11(2):259.

14. Qasim SB, Rehman IU. Application of nanomaterials in dentistry. In: Jackson M., Ahmed W. (eds) Micro and nanomanufacturing Volume II. Springer, Cham. 2018.

15. Padovani GC, Feitosa VP, Sauro S, Tay FR, Durán G, Paula AJ, Durán N. Advances in dental materials through nanotechnology: Facts, perspectives and toxicological aspects. Trends Biotech. 2015;33(11):621-636.

16. Sharan J, Singh S, Lale SV, Mishra M, Koul V, Kharbanda P. Applications of nanomaterials in dental science: A review. J Nanosci Nanotech. 2017;17(4):2235-2255.

17. Sakr OM, Alhablain EA. Assessment of the knowledge and attitude of dental students and intern practitioners about the nanotechnology in dentistry at KSA. IAIM. 2018;5(10):87-94.

18. Xenaki V, Marthinussen MC, Costea DE, Didilescu AC, Susin C, Cimpan MR, Åstrøm AN. Knowledge about nanotechnology and intention to use nanomaterials: A comparative study among dental students in Norway and Romania. Eur J Dent Educ. 2020;24(1):79-87.

19. Cormick C. Why Do We Need to Know What the Public Thinks about Nanotechnology? Nanoethics. 2009;3:167-173.

20. Kim Y, Lee EJ, Park SH, Kwon HJ, A An SS, Son SW, Seo YR, Pie J, Yoon M, Kim JH, Kim M. Comparative analysis of nanotechnology awareness in consumers and experts in South Korea. Int $\mathrm{J}$ Nanomed. 2014;9(2): 21-27.

21. Ipek Z, Atik A, Tan Ş, Erkoç F. Awareness, exposure, and knowledge levels of science teachers about nanoscience and nanotechnology. Issues Educ Res. 2020;30(1): 134-155.

22. Senocak E. A survey on nanotechnology in the view of the Turkish public. Sci Tech Soc. 2014;19:7994

23. Kohler AR, Som C. Environmental and health implications of nanotechnology-Have innovators learned the lessons from past experiences? Hum Ecol Risk Assess. 2008;14(3):512-531.

24. Cobb MD, Macoubrie J. Public perceptions about nanotechnology: Risks, benefits and trust. J Nanopartic Res. 2004;6(4):395-405. 
25. Muthukumar A, Duraisamy F, Ganapathy D. Awareness on nanoparticles incorporation in dental materials among undergraduate students-A cross sectional survey. J Pharm Res Int. 2020;32(16):63-74. 